\title{
Trueperella pyogenes and Brucella abortus Coinfection in a Dog and a Cat on a Dairy Farm in Egypt with Recurrent Cases of Mastitis and Abortion
}

\author{
Gamal Wareth $\mathbb{D}^{1,},{ }^{1,2}$ Mohamed El-Diasty, ${ }^{3}$ Falk Melzer, ${ }^{1}$ Jayaseelan Murugaiyan, ${ }^{4}$ \\ Amir Abdulmawjood $\mathbb{D}^{5},{ }^{5}$ Lisa D. Sprague, ${ }^{1}$ and Heinrich Neubauer ${ }^{1}$ \\ ${ }^{1}$ Friedrich-Loeffler-Institut, Federal Research Institute for Animal Health, Institute of Bacterial Infections and Zoonoses, \\ Naumburger Str. $96 a, 07743$ Jena, Germany \\ ${ }^{2}$ Department of Pathology, Faculty of Veterinary Medicine, Benha University, Moshtohor, Toukh 13736, Egypt \\ ${ }^{3}$ Animal Health Research Institute-Mansoura Provincial Laboratory, Mansoura, Egypt \\ ${ }^{4}$ Institute of Animal Hygiene and Environmental Health, Centre for Infectious Medicine, Freie Universität Berlin, \\ Robert-von-Ostertag-Str. 7-13, 14163 Berlin, Germany \\ ${ }^{5}$ Institute of Food Quality and Food Safety, Research Center for Emerging Infections and Zoonoses, \\ University of Veterinary Medicine Hannover, Bünteweg 17, 30559 Hannover, Germany
}

Correspondence should be addressed to Gamal Wareth; gamalwareth@hotmail.com

Received 19 September 2017; Accepted 11 February 2018; Published 20 March 2018

Academic Editor: Giuliano Bettini

Copyright (C) 2018 Gamal Wareth et al. This is an open access article distributed under the Creative Commons Attribution License, which permits unrestricted use, distribution, and reproduction in any medium, provided the original work is properly cited.

Trueperella pyogenes was isolated from a dog and a cat with a mixed infection with Brucella abortus. Both lived on a dairy cattle farm with a history of regular cases of abortion and mastitis. Identification of the bacteria was done by means of MALDI-TOF MS, loopmediated isothermal amplification (LAMP) based on cpn60, partial 16S rRNA sequencing, and growth on Loeffler Serum Medium. Isolation of Trueperella pyogenes on the dairy farm highlights its neglected role in reproduction failure and draws attention to its effects in the dairy industry in Egypt. Diagnosis and control of abortion in Egypt should include Trueperella pyogenes as one of possible causes of abortion.

\section{Introduction}

Trueperella (T.) pyogenes is Gram positive, haemolytic, nonmotile, non-spore-forming, facultative anaerobic coccobacillus. The species was originally known as Corynebacterium pyogenes and Actinomyces pyogenes and then as Arcanobacterium pyogenes. Based on the $16 \mathrm{~S}$ rRNA signature nucleotide comparisons and menaquinone and phospholipid compositions, the genus was divided into Arcanobacterium and Trueperella gen. nov., in honour of the German microbiologist Hans G. Trüper. Currently, the genus Trueperella encompasses five different species, which are, $T$. pyogenes, $T$. abortisuis, T. bonsai, T. bernardiae, and T. bialowiezensis [1].

Trueperella pyogenes is a commensal and an opportunistic pathogen which can cause a variety of suppurative infections in livestock and wildlife and can elicit sporadic cases of abortion due to endometritis and placentitis at any stage of gestation [2]. The bacteria can affect a wide range of animal species, including cattle, camel, horse, swine, antelope, bison, chicken, pigeon, turkey, deer, elephant, gazelle, macaw, and reindeer [3], as well as companion animals such as dogs and cats [4]. T. pyogenes has recently been isolated from lung abscesses of slaughtered one-humped camels in Cairo presenting with clinical and subclinical pulmonary infection [5]. Different suppurative T. pyogenes infections in livestock and companion animals are associated with a variety of virulence factors, particularly exotoxin pyolysin and adhesins (fimbriae, neuraminidases, and collagen-binding protein) [6].

The present study describes the isolation of $T$. pyogenes in the uterine discharge of a bitch after abortion and from a cat presenting with pyometra. Both animals lived on a dairy 
farm with recurrent cases of abortion and mastitis due to Brucella (B.) abortus biovar (bv) 1 infections [7]. The aim of the current study was to identify and highlight a neglected pathogen in Egypt which can circulate in pet animals in contact with livestock and cause reproductive failure within the dairy industry.

\section{Material and Methods}

2.1. Bacteriology. Uterine discharges were collected from the bitch and the cat under sterile conditions after positive Brucella serology using Rose Bengal Test and Complement Fixation Test and sent to the OIE reference laboratory of brucellosis at the Friedrich-Loeffler-Institut for Brucella identification and biotyping. During cultivation on blood agar for Brucella biotyping, two types of bacterial colonies were found. Identification and biotyping of the putative Brucella isolates were done by assessing colony morphology, biochemical reactions, $\mathrm{CO}_{2}$ requirement, production of $\mathrm{H}_{2} \mathrm{~S}$, growth in the presence of the dyes thionine and fuchsine, reaction with monospecific antisera ( $A, M$, and $\mathrm{R}$ ), and phage lysis ( $\mathrm{Wb}, \mathrm{Tb}$, and F25) [8]. Putative Trueperella strains were additionally grown on Loeffler Serum Medium (Himeda, Mumbai, India) and tested for catalase activity.

2.2. MALDI Based Species Identification. The microbial species identification was carried out using matrix-assisted laser desorption/ionization (MALDI-TOF MS) as described elsewhere [9]. In brief, bacteria from single colonies were inactivated by the addition of $300 \mu \mathrm{L}$ of HPLC grade water and $900 \mu \mathrm{L}$ of absolute ethanol. For protein extraction, the suspensions were centrifuged at $11290 \mathrm{~g}$ for $2 \mathrm{~min}$, the supernatants discarded, and the resulting cell pellets air-dried to remove traces of ethanol. Each pellet was reconstituted in $50 \mu \mathrm{L}$ of $70 \%$ formic acid and $50 \mu \mathrm{L}$ of acetonitrile. The samples were then sonicated (100\% amplitude and 1.0 duty cycle) for $1 \mathrm{~min}$ on ice using a sonicator (UP100H; Hielscher Ultrasound Technology, Teltow, Germany). Next, samples were centrifuged at $11290 \mathrm{~g}$ for $5 \mathrm{~min}$ at room temperature and the clear supernatant was collected. One $\mu \mathrm{L}$ of each supernatant was spotted onto the MALDI target (MSP 96 target polished steel (MicroScout Target) plate; Bruker Daltonik, Bremen, Germany), air-dried, and overlaid with $1.0 \mu \mathrm{L}$ of saturated $\alpha$-cyano-4-hydroxycinnamic acid matrix solution (in $50 \%$ acetonitrile and $0.25 \%$ trifluoroacetic acid). The MALDI measurements were carried out using a Microflex LT (Bruker Daltonics, Bremen, Germany) instrument and MBT Compass Explorer 4.1 software (Bruker Daltonics, Bremen, Germany). The MALDI Biotyper manufacturer's recommendation on log score value of $0-3$ for species identification was followed. Score values between 2.3 and 3.0 indicate "highly probable species identification"; values between 2.0 and 2.29 indicate a "secure genus identification and probable species identification"; values between 1.7 and 1.99 indicate "probable genus identification"; and values between 0 and 1.69 indicate "no reliable identification."
2.3. DNA Extraction, AMOS-PCR, and Partial 16S rRNA Sequencing. Genomic DNA was extracted from heat inactivated individual bacterial colonies using the High Pure PCR template preparation kit (Roche Applied Sciences, Mannheim, Germany) according to the manufacturer's instructions. AMOS-PCR for B. abortus, B. melitensis, $B$. ovis, and $B$. suis was done as previously described [7, 10]. Partial 16S rRNA genes of the bacterial isolates were amplified by PCR with the 16SUNI-L ( $5^{\prime}$-AGA GTT TGA TCA TGG CTC AG- $3^{\prime}$ ) and 16SUNI-R ( $5^{\prime}$-GTG TGA CGG GCG GTG TGT AC- $3^{\prime}$ ) primer pair (Jena Bioscience $\mathrm{GmbH}$, Jena, Germany) to generate amplicons of approx. 1,400-bp [11]. PCR products were analyzed by agarose gel electrophoresis, bands were cut out, and DNA was purified using a Gel Extraction Kit (Qiagen, Hilden, Germany) according to the manufacturer's recommendations. Cycle sequencing of the partial 16S rRNA genes was done in both directions by using forward and reverse amplification primers with a BigDye Terminator Version 1.1 Cycle Sequencing Kit (Applied Biosystems, Darmstadt, Germany) according to the recommendations of the manufacturer. Sequencing products were analyzed with an ABI Prism 3130 Genetic Analyzer (Applied Biosystems). Identification of isolates was done by a BLAST search (https://www.ncbi.nlm.nih.gov/blast/) using 16S rRNA gene sequences.

2.4. Loop-Mediated Isothermal Amplification (LAMP). A newly designed loop-mediated isothermal amplification (LAMP) assay based on cpn60 (encoding for chaperonin 60) was carried out in a total volume of $25 \mu \mathrm{L}$ containing $0.5 \mu \mathrm{L}$ each of pho-F3 and Pyo-B3 primer $(25 \mathrm{pmol} / \mu \mathrm{L})$ equivalent to $0.5 \mu \mathrm{M}$ final concentration, $1 \mu \mathrm{L}$ each of Pyo-LoopF and Pyo-LoopB primer $(25 \mathrm{pmol} / \mu \mathrm{L})$ equivalent to $1.0 \mu \mathrm{M}$ final concentration, $2 \mu \mathrm{L}$ each of Pyo-FIP and Pyo-BIP primer ( $25 \mathrm{pmol} / \mu \mathrm{L}$ ) equivalent to $2.0 \mu \mathrm{M}$ final concentration, and $15 \mu \mathrm{L}$ Isothermal Master Mix Iso-001 (Optigene, Horsham, UK). Subsequently, $3 \mu \mathrm{L}$ DNA was added as a template. The LAMP assay was run at $70^{\circ} \mathrm{C}$ for $30 \mathrm{~min}$ with a melting curve analysis step (annealing curve $98^{\circ} \mathrm{C}$ to $80^{\circ} \mathrm{C}$ ramping at $0.05^{\circ} \mathrm{C}$ per s) in a portable real-time fluorometer (Genie II ${ }^{\circledR}$, Optigene, West Sussex, UK) according to the manufacturer's instructions as previously described [12].

\section{Results and Discussion}

In addition to $B$. abortus bvl, a Gram positive, catalase negative, aerobic, nonmotile, $ß$-haemolytic bacterium was recovered from a bitch who had recently aborted and from a cat suffering from an open pyometra. Both animals lived on a dairy farm with regular cases of abortion and mastitis [7]. These recovered bacteria produced $\mathrm{H}_{2} \mathrm{~S}$ and were able to grow under aerobic conditions with and without $\mathrm{CO}_{2}$. They did not react with the tested monospecific antisera and phages specific for Brucella and produced no amplicon in the AMOS-PCR. MALDI-TOF MS identified these bacteria as T. pyogenes with a log score of 2.18 (dog isolate) and a log score of 2.02 (cat isolate), respectively. These log score values confirm the identification at the species level. MALDI-TOF 
TABLE 1: Results of LAMP including detection time and annealing temperature of each isolate and positive and negative control.

\begin{tabular}{lcccc}
\hline Number & Isolate number 1 & Isolate number 2 & Positive control & Negative control \\
\hline Sample ID & 15RB7429H & 15RB7430H & T. pyogenes DSM 20594 & HPLC water and Master mix \\
Result & $+\mathrm{ve}$ & $+\mathrm{ve}$ & $+\mathrm{ve}$ & $-\mathrm{ve}$ \\
Detection time & $10: 00$ & $7: 30$ & $17: 30$ & 00 \\
Annealing & 89.7 & 89.6 & 89.6 & 00 \\
\hline
\end{tabular}

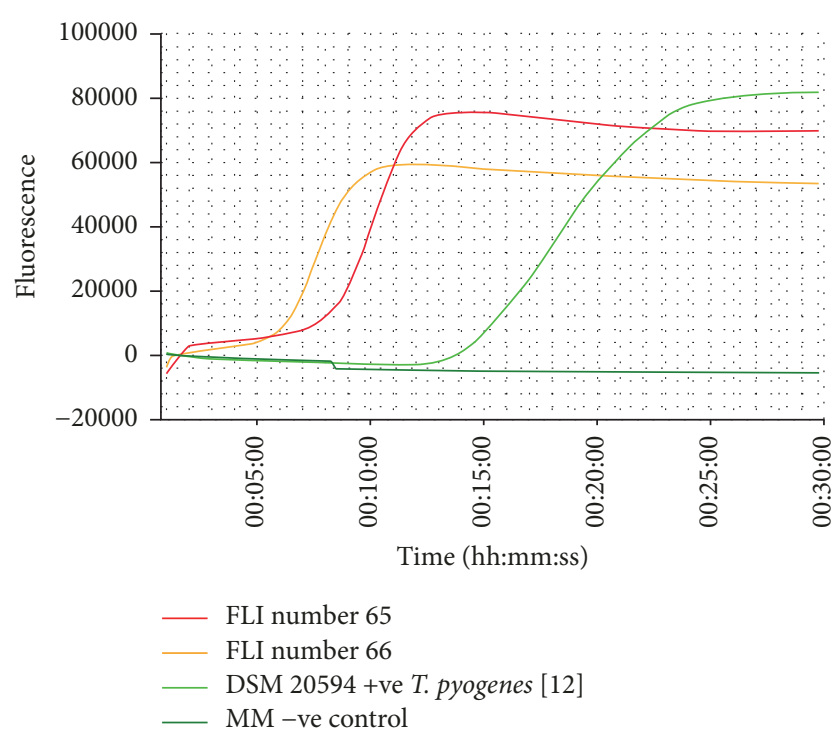

FIGURE 1: Loop amplification signal of the LAMP products of the two T. pyogenes isolates, the reference strain T. pyogenes DSM 20594, and the negative control.

MS for Trueperella differentiation with a log score around 2 shows comparable discriminating power with molecular methods; it is a rapid and accurate tool for T. pyogenes diagnosis [13]. Partial $16 \mathrm{~S}$ rRNA sequencing confirmed the isolates to be T. pyogenes and cultivation on Loeffler serum revealed the typical pitting of the serum slope. The species was also identified using the cpn60 LAMP assay. A loop amplification signal of the LAMP products was observed for both $T$. pyogenes isolates and for the reference strain T. pyogenes DSM 20594 and none in the negative control containing water and LAMP-Mastermix (Figure 1). The mean of the annealing temperature of the amplicons was $89,6^{\circ} \mathrm{C}$ sd $\pm 0.05 \mathrm{~s}$ (Figure 2). The cpn60 LAMP assay allowed a reliable, rapid, and low cost identification of T. pyogenes (Table 1).

The chaperonin 60 encoding gene has been previously used for the identification of various Gram positive bacteria and a chaperonin sequence database containing a large collections of sequences including gene cpn60 of T. pyogenes is available. This was comparable to the previously described LAMP-mediated identification of A. pluranimalium using pla gene [14].

Trueperella pyogenes is a ubiquitous occurring organism and is frequently found as a commensal in the oropharynx, upper respiratory tract, and gastrointestinal tract of livestock [2]. However, underlying chronic illness, innate immunity, poor animal husbandry, and production methods appear to

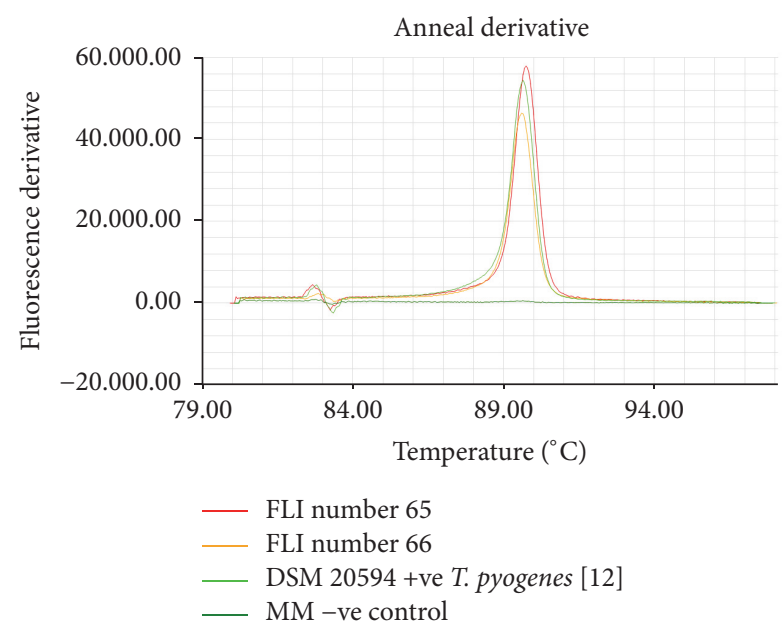

FIGURE 2: Annealing reaction curves of the respective amplicons.

influence the virulence of the agent [15-18]. It can readily be transmitted by biting flies and contaminated farm and dairy equipment [6].

In Egypt, the dairy industry suffers from large financial losses due to reduced fertility and milk yield as a consequence of uterine infections and mastitis caused not only by brucellosis [5] but also by other agents such as E. coli, Fusobacterium necrophorum, and T. pyogenes $[19,20]$. Although T. pyogenes is a well-known agent causing reproductive disorders in male and female livestock species [4], its role is neglected as primary cause of abortion in cattle, as diagnosis of abortion is focusing only on predominately classical agents, such as Brucella species. Circulating of the bacterium in dog and cat kept in dairy farm with history of abortion and mastitis is alarming and representing a potential reservoir of infection for livestock. Thus screening for this agent should be considered in cases of seronegativity to Brucella. On the other hand, eradication of pet animals has to be implemented and taken into account in control measures to reduce dissemination of pathogens. T. pyogenesis is not a sufficiently known pathogen in animals and humans because of inadequate identification of this bacterium that should be better known to clinical microbiologists. Diagnosis of abortion in cattle and control has to include T. pyogenesis especially in farm suffering from mastitis and sporadic abortion.

\section{Disclosure}

This manuscript has not been submitted for publication elsewhere and has been approved by all coauthors. 


\section{Conflicts of Interest}

The authors declare that they do not have any conflicts of interest.

\section{Acknowledgments}

The authors would like to acknowledge the Academy of Scientific Research and Technology (ASRT), Egypt, for financial support. This work belongs to BrucMedNet project (ID: 698) funded by the ARIMNet2.

\section{References}

[1] A. F. Yassin, H. Hupfer, C. Siering, and P. Schumann, "Comparative chemotaxonomic and phylogenetic studies on the genus Arcanobacterium Collins et al. 1982 emend. Lehnen et al. 2006: proposal for Trueperella gen. nov. and emended description of the genus Arcanobacterium," International Journal of Systematic and Evolutionary Microbiology, vol. 61, no. 6, pp. 1265-1274, 2011.

[2] B. H. Jost and S. J. Billington, "Arcanobacterium pyogenes: molecular pathogenesis of an animal opportunist," Antonie van Leeuwenhoek, vol. 88, no. 2, pp. 87-102, 2005.

[3] S. J. Billington, K. W. Post, and B. H. Jost, "Isolation of Arcanobacterium (Actinomyces) pyogenes from cases of feline otitis externa and canine cystitis," Journal of Veterinary Diagnostic Investigation, vol. 14, no. 2, pp. 159-162, 2002.

[4] M. G. Ribeiro, R. M. Risseti, C. A. D. Bolaños et al., "Trueperella pyogenes multispecies infections in domestic animals: a retrospective study of 144 cases (2002 to 2012)," Veterinary Quarterly, vol. 35, no. 2, pp. 82-87, 2015.

[5] G. Wareth, J. Murugaiyan, D. F. Khater, and S. A. Moustafa, "Subclinical pulmonary pathogenic infection in camels slaughtered in Cairo, Egypt," The Journal of Infection in Developing Countries, vol. 8, no. 7, pp. 909-913, 2014.

[6] R. M. Risseti, E. Zastempowska, M. Twarużek et al., "Virulence markers associated with Trueperella pyogenes infections in livestock and companion animals," Letters in Applied Microbiology, vol. 65 , no. 2 , pp. 125-132, 2017.

[7] G. Wareth, F. Melzer, M. El-Diasty et al., "Isolation of Brucella abortus from a dog and a cat confirms their biological role in re-emergence and dissemination of bovine brucellosis on dairy farms," Transboundary and Emerging Diseases, vol. 64, no. 5, pp. e27-e30, 2017.

[8] G. G. Alton, L. M. Jones, R. D. Angus, and J. M. Verger, Techniques for the Brucellosis Laboratory, Instituttional de la Recherche Agronomique, Paris, France, 1988.

[9] J. Murugaiyan, B. Walther, I. Stamm et al., "Species differentiation within the Staphylococcus intermedius group using a refined MALDI-TOF MS database," Clinical Microbiology and Infection, vol. 20, no. 10, pp. 1007-1015, 2014.

[10] B. J. Bricker and S. M. Halling, "Differentiation of Brucella abortus bv. 1, 2, and 4, Brucella melitensis, Brucella ovis, and Brucella suis bv. 1 by PCR," Journal of Clinical Microbiology, vol. 32, no. 11, pp. 2660-2666, 1994.

[11] P. Kuhnert, S. E. Capaul, J. Nicolet, and J. Frey, "Phylogenetic positions of Clostridium chauvoei and Clostridium septicum based on 16S rRNA gene sequences," International Journal of Systematic Bacteriology, vol. 46, no. 4, pp. 1174-1176, 1996.
[12] A. Abdulmawjood, J. Wickhorst, O. Hashim et al., "Application of a loop-mediated isothermal amplification (LAMP) assay for molecular identification of Trueperella pyogenes isolated from various origins," Molecular and Cellular Probes, vol. 30, no. 4, pp. 205-210, 2016.

[13] L. Z. Moreno, C. E. C. Matajira, B. L. P. da Costa et al., "Characterization of porcine Trueperella pyogenes by matrix-assisted laser desorption ionization-time of flight mass spectrometry (MALDI-TOF MS), molecular typing and antimicrobial susceptibility profiling in Sao Paulo State," Comparative Immunology, Microbiology \& Infectious Diseases, vol. 51, pp. 49-53, 2017.

[14] A. Abdulmawjood, J. Wickhorst, O. Sammra et al., "Development of a loop-mediated isothermal amplification (LAMP) assay for rapid and sensitive identification of Arcanobacterium pluranimalium," Molecular and Cellular Probes, vol. 29, no. 6, pp. 468-472, 2015.

[15] N. Dyer, K. B. Register, D. Miskimins, and T. Newell, "Necrotic pharyngitis associated with Mycoplasma bovisinfections in American bison (Bison bison)," Journal of Veterinary Diagnostic Investigation, vol. 25, no. 2, pp. 301-303, 2013.

[16] J. S. Agerholm, T. K. Jensen, J. F. Agger, M. Y. Engelsma, and H. I. J. Roest, "Presence of Coxiella burnetii DNA in inflamed bovine cardiac valves," BMC Veterinary Research, vol. 13, no. 1, article 69, 2017.

[17] L. C. Carneiro, J. G. Cronin, and I. M. Sheldon, "Mechanisms linking bacterial infections of the bovine endometrium to disease and infertility," Reproductive Biology, vol. 16, no. 1, pp. $1-7,2016$

[18] P. H. Kvist, E. S. Jensen, B. Aalbaek, and H. E. Jensen, "Evaluation of the pathology, pathogenesis and aetiology of auricular elephantiasis in slaughter pigs," Journal of Veterinary Medicine Series A. Physiology, Pathology, Clinical Medicine, vol. 49, no. 10, pp. 517-522, 2002.

[19] H. El-Khadrawy, W. Ahmed, M. Zaabal, and E. Hanafi, "Strategies for diagnosis and treatment of uterine infection in bovines," Global Veterinaria, vol. 15, no. 5, pp. 98-105, 2015.

[20] M. L. S. Bicalho, F. S. Lima, V. S. Machado et al., "Associations among Trueperella pyogenes, endometritis diagnosis, and pregnancy outcomes in dairy cows," Theriogenology, vol. 85, no. 2, pp. 267-274, 2016. 

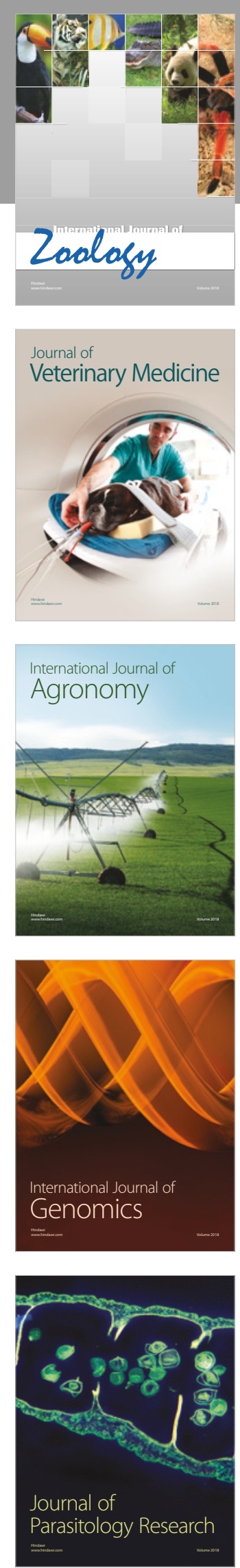

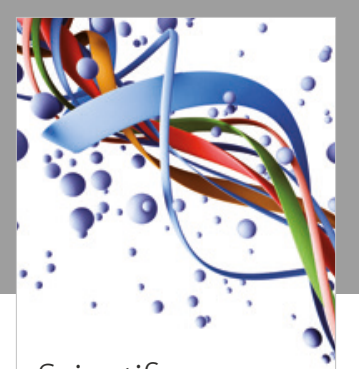

Case Reports in Veterinary Medicine Scientifica
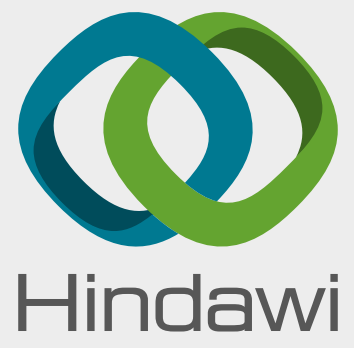

Submit your manuscripts at

www.hindawi.com
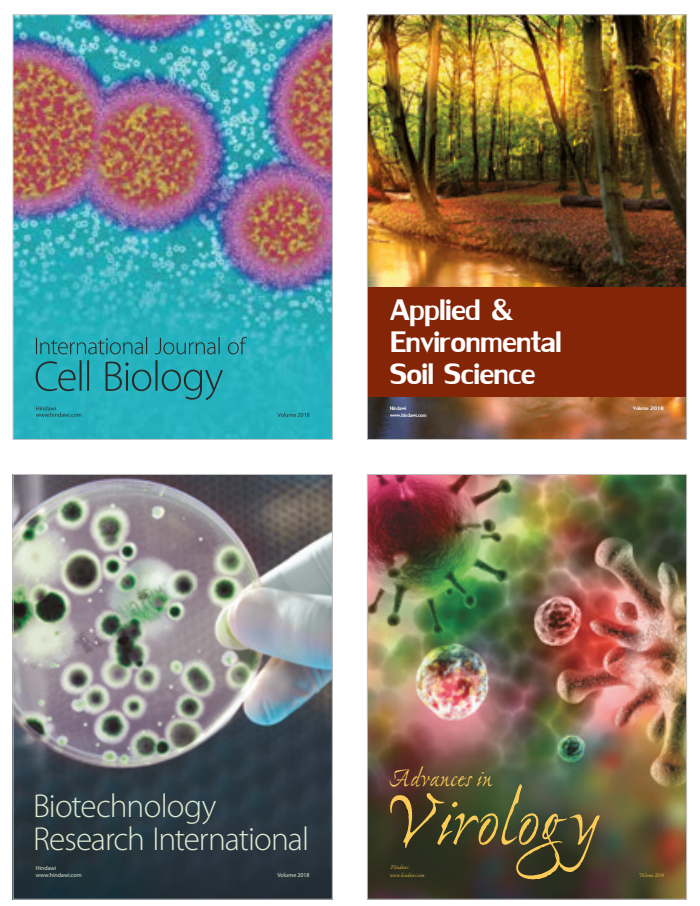

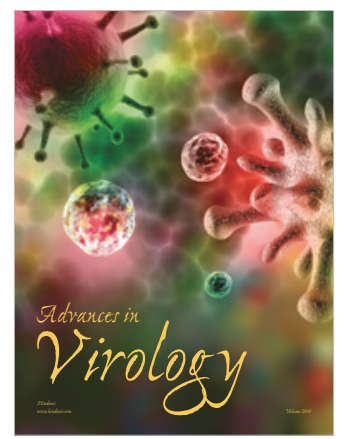

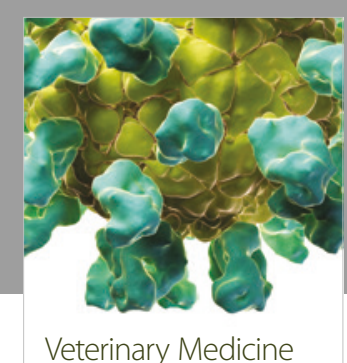
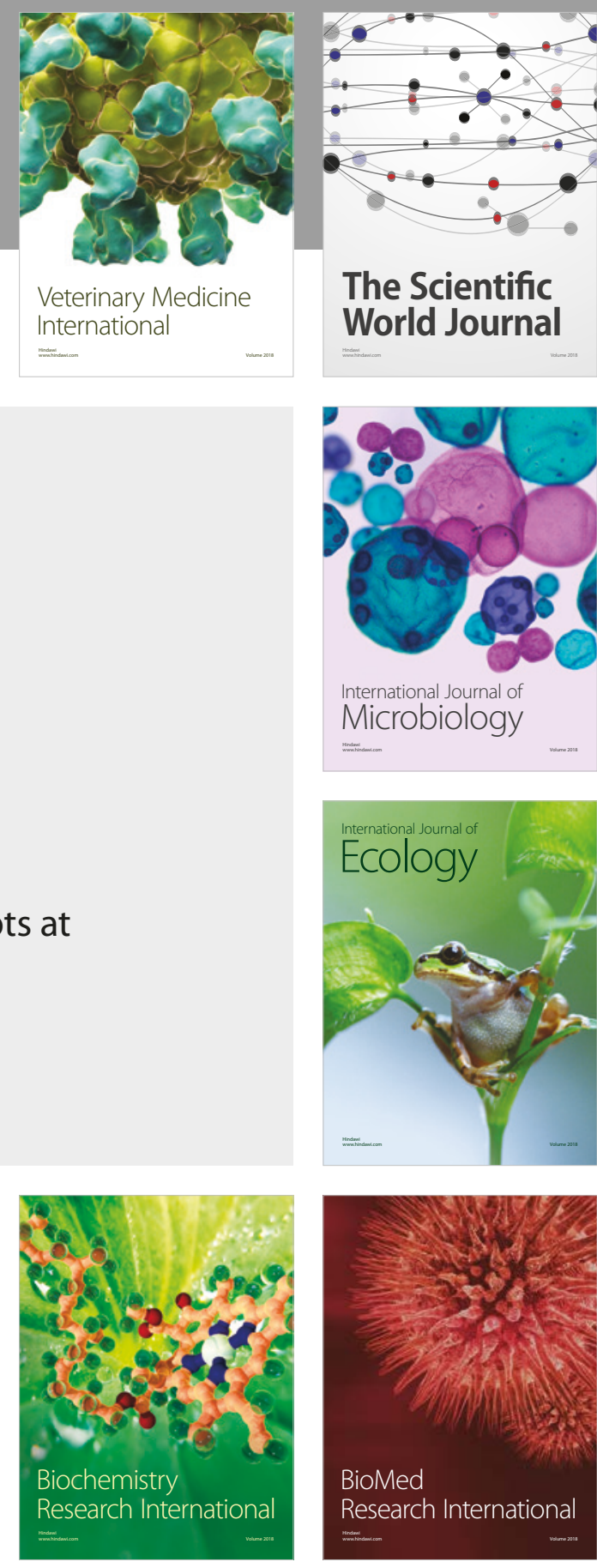

The Scientific World Journal

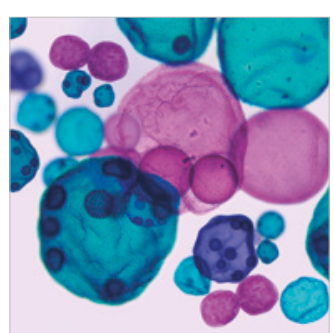

International Journal of Microbiology
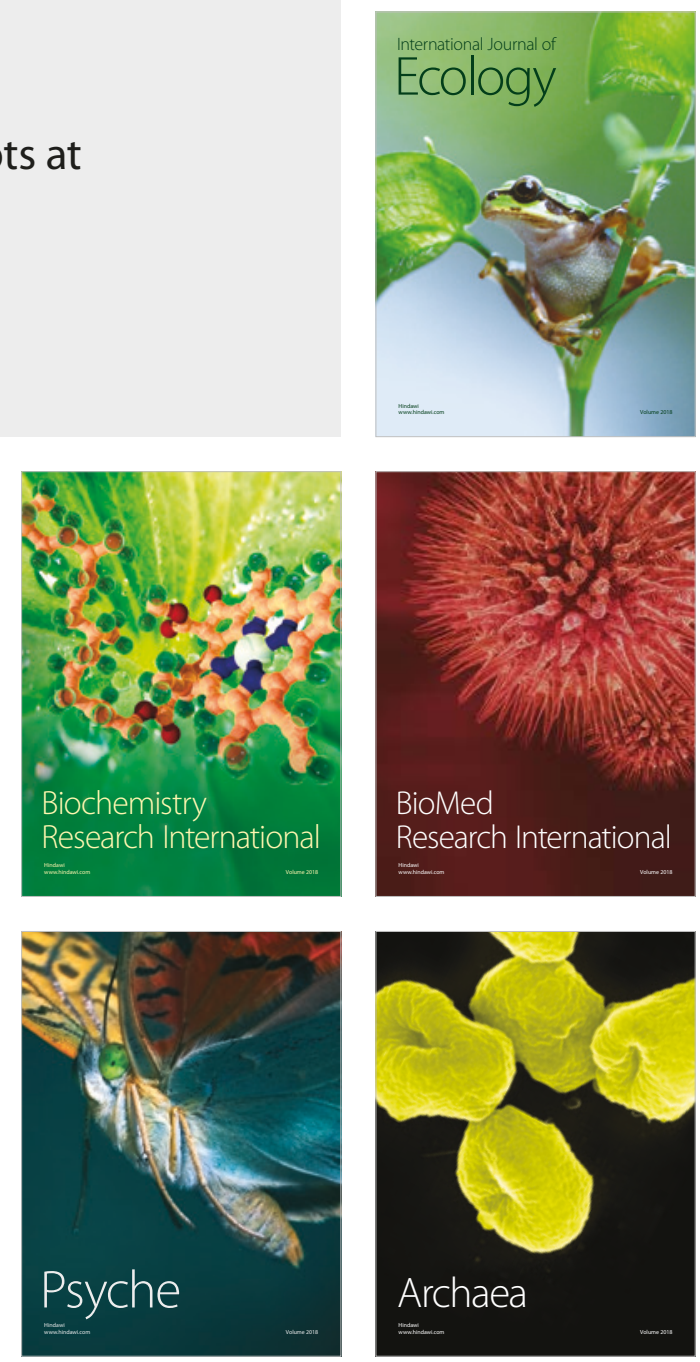